\title{
The antidepressant effect of drugs depends on the pain and stress experienced at early stages of development
}

\author{
Butkevich Irina $\mathrm{P}^{*}$, Mikhailenko Viktor A \\ I.P. Pavlov Institute of Physiology RAS, Saint-Petersburg, Russia
}

Selective serotonin reuptake inhibitor fluoxetine is recommended for pregnant women to treat depression during pregnancy [1]. The 5-hydroxytryptamine 1A (5-HT1A) receptor agonist buspirone is widely used for treating generalized anxiety disorder, in certain conditions buspirone can show antidepressant effect [2]. Both drugs act through the 5-HT1A receptor, which is the target of prenatal stress and antidepressants [2,3]. This type of receptor is widely represented in the central nervous system, especially in structures involved in psycho-emotional behavior, stressful behavior and nociception, which determines the close functional relationship between the serotoninergic system and the HPA axis and nociceptive system [4]. Various types of stressors can alter antidepressant action of these drugs. In this study, we investigated a previously unexplored question: how will be changed the effects of fluoxetine and buspirone, introduced to dam rats during pregnancy, on the adaptive behavior of the offspring subjected to prenatal stress (stress of pregnant dams) and inflammatory pain in the first two days of life.

Wistar male and female rats were obtained from the biocollection of I.P. Pavlov Institute of Physiology of the RAS and maintained in the laboratory vivarium under standard conditions. Pregnancy was determined on the following day after mating. The present study was approved by Animal Care and Use Committee of the I.P. Pavlov Institute of Physiology, the Russian Academy of Sciences (St. Petersburg, Russia) and followed the guidelines published by the Committee for Research and Ethical Issues of the IASP on ethical standards for investigations of experimental pain in animals. Intraperitoneal injection of antidepressant fluoxetine $(10 \mathrm{mg} / \mathrm{kg}$, i.p.) or anxiolytic buspirone $(3.5 \mathrm{mg} / \mathrm{kg}$, i.p.) was given to Wistar dam rats from day 9 to day 20 of pregnancy; from day 15 to delivery the rats were exposed to restraint stress, appropriate controls were also used. Here, fluoxetine and buspirone were injected to rat dams which were stressed during pregnancy, not stressed rat dams with injection of these drugs were studied previously. Restraining stress was carried out in the morning and evening (at different times) during an hour in bright light. Newborn offspring was subjected to peripheral inflammatory pain (the subcutaneous injection of $2.5 \%$ formalin 0.5 $\mu \mathrm{l}$ in the sole of the left hind paw) on the first day and repeatedly the second day after birth.

From the age of 25 days, basal pain sensitivity in the hot plate test, the inflammatory pain response in the formalin test, the levels of anxiety and depression in the elevated plus maze and forced swim test, and the spatial learning ability in the Morris water maze were investigated. In all of the behavioral paradigms prenatal stress worsened behavioral indices as compared to indices of control animals: lowered pain threshold on the hot plate, strengthened the inflammatory pain response in the formalin test, decreased the time spent on the open arms in the elevated plus maze, increased the immobility time in the forced swim test and the time of the platform searching in the Morris water maze. Chronic injection of fluoxetine or buspirone to pregnant stressed dams improved all behavioral indices. Inflammatory pain in newborns strengthened inflammatory pain response integrated at the supraspinal level in the formalin test and increased the level of depressive-like behavior in the adolescent rats, but did not alter the behavior in the rest tests. Pain in prenatally stressed newborns in none of the tests strengthened the negative effect of prenatal stress. Moreover, pain in early life inhibited the pronociceptive effect of prenatal stress in the hot plate test and the formalin test and normalized pain sensitivity and inflammatory pain response. It resulted in neutralization of antinociceptive effects of fluoxetine and buspirone. Our data provide evidence that fluoxetine and buspirone are able to penetrate placental barrier and can induce long-term antinociceptive effect in the offspring. Antinociceptive, anxiolytic and antidepressive effects of fluoxetine and buspirone are obvious only in rats with algesia, or anxiety-depressive behavior. The present data suggest that the interaction of two types stress during the critical periods of development can program adaptive behavior to pain stress in later life what supports the match/mismatch hypothesis $[5,6]$. Our previous data demonstrated that adult rats, which were exposed to short-term maternal deprivation at once after formalin-induced pain in the first two days of life, showed alleviated pain response in the formalin test as compared to hyperalgesic response in adult rats that experienced only pain without maternal deprivation [7]. The fact that one negative stress can alleviate the consequences of another negative event what is manifested in the formation of a positive phenotype and the enhancement of adaptive abilities to stress in later life is the most important conclusion from our two studies. The mechanism of this phenomenon is intensively investigated.

\section{Funding}

The study was supported by the Russian Foundation for Basic Research (grant no. 17-04-00214-a).

\section{References}

1. Kaihola H, Yaldir FG, Hreinsson J, Hornaeus K, Bergquist J, Olivier JDA, et al. (2016) Effects of fluoxetine on human embryo development. Front Cell Neurosci 10: 160.

2. Albert PR, Fiori LM (2014) Transcriptional dys-regulation in anxiety and major depression: 5-HT1A gene promoter architecture as a therapeutic opportunity. Curr Pharm 20: 3738-3750.

*Correspondence to: Butkevich Irina P, I.P. Pavlov Institute of Physiology RAS, Saint-Petersburg, Russia, E-mail: irinabutkevich@yandex.ru

Received: May 02, 2018; Accepted: May 21, 2018; Published: May 24, 2018 
3. Kiryanova V, Smith VM, Antle MC, Dyck RH (2018) Behavior of Adult 5-HT1 Receptor Knockout Mice Exposed to Stress During Prenatal Development. Neurosci 371: $16-28$

4. Lanfumey L, Mongeau R, Cohen-Salmon C, Hamon M (2008) Corticosteroid-serotonin interactions in the neurobiological mechanisms of stress-related disorders. Neurosci Biobehav Rev 32: 1174-1184.

5. Nederhof E, Schmidt MV (2012) Mismatch or cumulative stress: Toward an integrated hypothesis of programming effects. Physiol Behav 106: 691-700.
6. Verstraeten BSE, McCreary J, Weyers S, Metz GAS, Olson DM (2019) Prenatal two-hit stress affects maternal and offspring pregnancy outcomes and uterine gene expression in rats: match or mismatch? Biol Reprod 100: 195-207.

7. Butkevich IP, Mikhailenko VA, Vershinina EA, Aloisi AM, Barr GA (2017) Long-Term Effects of Chronic Buspirone during Adolescence Reduce the Adverse Influences of Neonatal Inflammatory Pain and Stress on Adaptive Behavior in Adult Male Rats. Front Behav Neurosci 26: 11.

Copyright: $(2018$ Butkevich Irina P. This is an open-access article distributed under the terms of the Creative Commons Attribution License, which permits unrestricted use, distribution, and reproduction in any medium, provided the original author and source are credited. 